\title{
An NMR-based metabolomic approach to assess metabolism in splanchnic tissues of steers
}

\author{
H.C. Bertram ${ }^{1}$, N.B. Kristensen ${ }^{2,5}$, A. Malmendal ${ }^{3}$, N.C. Nielsen ${ }^{3}$, \\ S.K. Jensen ${ }^{2}$ and D.L. Harmon ${ }^{4}$ \\ ${ }^{1}$ Department of Food Science, \\ ${ }^{2}$ Department of Animal Nutrition and Physiology, \\ Danish Institute of Agricultural Sciences \\ Box 50, DK-8830 Tjele, Denmark \\ ${ }^{3}$ Interdisciplinary Nanoscience Center (iNANO), Department of Chemistry, \\ University of Aarhus \\ Langelandsgade 140, DK-8000 Aarhus C, Denmark \\ ${ }^{4}$ Department of Animal Sciences, University of Kentucky \\ Lexington, KY 40546, USA
}

\begin{abstract}
${ }^{1} \mathrm{H}$ NMR spectra were acquired on plasma samples obtained from the mesenteric artery, hepatic portal vein and the hepatic vein of steers $(n=6)$ to demonstrate a NMR-based metabolomic approach to study splanchnic metabolism. The metabolic differences between the different plasma samples (arterial, portal, hepatic) were examined by partial least square regressions (PLSR) on the obtained spectra. These analyses revealed spectral differences that could be ascribed to changes in the level of propionate, $\beta$-hydroxybutyrate, lactate, acetate, $n$-butyrate and glucose, which was supported by biochemical analyses.
\end{abstract}

KEY WORDS: NMR, metabolomics, splanchnic, metabolism, steer, plasma

\section{INTRODUCTION}

Evolving modeling of animal performance by simulation of fermentation, digestion, absorption, and metabolism poses a large demand for detailed experimental data to describe biological relationships in metabolism and for validation of simulation models. The global nature of the models demands identification and quantification of a large number of metabolites in blood and

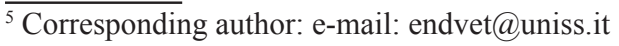


blood plasma samples that will be very tedious to obtain by traditional chemical methods. The objective of the present study was to make an initial evaluation of high-resolution ${ }^{1} \mathrm{H}$ NMR spectroscopy as a tool to investigate a large range of inter-organ fluxes of low molecular and hydrophilic metabolites in splanchnic tissues.

\section{MATERIAL AND METHODS}

Thirty-six blood plasma samples (arterial, portal and hepatic) stabilized with sodium heparin were obtained from the study of Kristensen and Harmon (2004). The washed rumen of six steers was incubated with a bicarbonate buffer without VFA for $45 \mathrm{~min}$ (Control samples) and a VFA-buffer as well as continuous intraruminal infusion of VFA for $4 \mathrm{~h}$ (VFA samples). The NMR measurements were performed at $300 \mathrm{~K}$ on a Bruker Avance $400 \mathrm{NMR}$ Spectrometer, operating at a ${ }^{1} \mathrm{H}$ frequency of $400.13 \mathrm{MHz}$, equipped with a 5-mm HX inverse probe. Prior to the measurements $400 \mu \mathrm{l}$ aliquots of the samples were combined with $200 \mu \mathrm{l}$ of a $\mathrm{D}_{2} \mathrm{O}$ solution containing $10 \mathrm{mg} \mathrm{TSP} / \mathrm{ml}$. One-dimensional spectra were acquired with a Carr-Purcell-Meiboom-Gill (CPMG) delay. The total CPMG delay was 40 $\mathrm{ms}$ and the spin-echo delay was $200 \mu \mathrm{s}$. The recycling delay was $2 \mathrm{sec}$, during which water presaturation was applied. A total of 128 transients of $8 \mathrm{~K}$ data points spanning a spectral width of $12.015 \mathrm{ppm}$ were collected. An exponential linebroadening function of $3 \mathrm{~Hz}$ was applied to the FID prior to FT. All spectra were referenced to the TSP signal at $0 \mathrm{ppm}$. The mean-normalized ${ }^{1} \mathrm{H}$ NMR spectra in the region $0.5-4.6 \mathrm{ppm}$ and the region 5.1-9 ppm corresponding to 5455 data points were used for further data analysis, which was performed using the Unscrambler Software version 8.0 (Camo, Oslo, Norway). Principal component analysis (PCA) was applied to the centered data to explore any clustering behaviour of the samples, and partial least square regression-discriminant analysis (PLS-DA) was performed to explore intrinsic biochemical dissimilarities between predefined sample classes.

\section{RESULTS AND DISCUSSION}

Figure 1 displays a typical ${ }^{1} \mathrm{H}$ spectrum obtained for an arterial plasma sample. Several resonance lines are detected which have tentatively been identified and are presented in the figure caption.

To examine the metabolite concentration differences between the different plasma samples (arterial, portal, hepatic), PCA and PLS-DA were performed on the obtained spectra. The loadings froma PLS-DA discriminating between arterial and portal plasma, which reveal those variables that are most important in separation of the two plasma types, are shown in Figure 2. The loadings highlight differences in the 


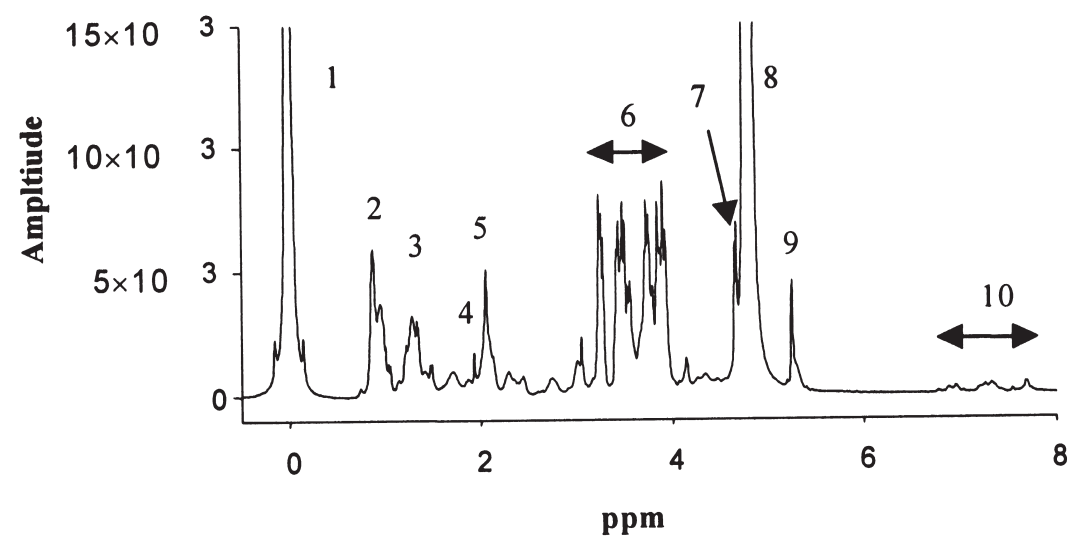

Figure 1. Example of ${ }^{1} \mathrm{H}$ NMR spectrum of an arterial plasma sample. The main resonances observed are assigned as follows: 1 TSP (standard), 2 lactate, 3 VLDL/LDL, 4 acetate, 5 lipid $\left(\mathrm{CH}_{2}-\mathrm{CH}_{2}-\mathrm{CH}=\right)$, 6 glucose and amino acid $\mathrm{CH}, 7 \beta$-glucose, $8 \mathrm{HDO}, 9 \alpha$-glucose, 10 aromatic compounds

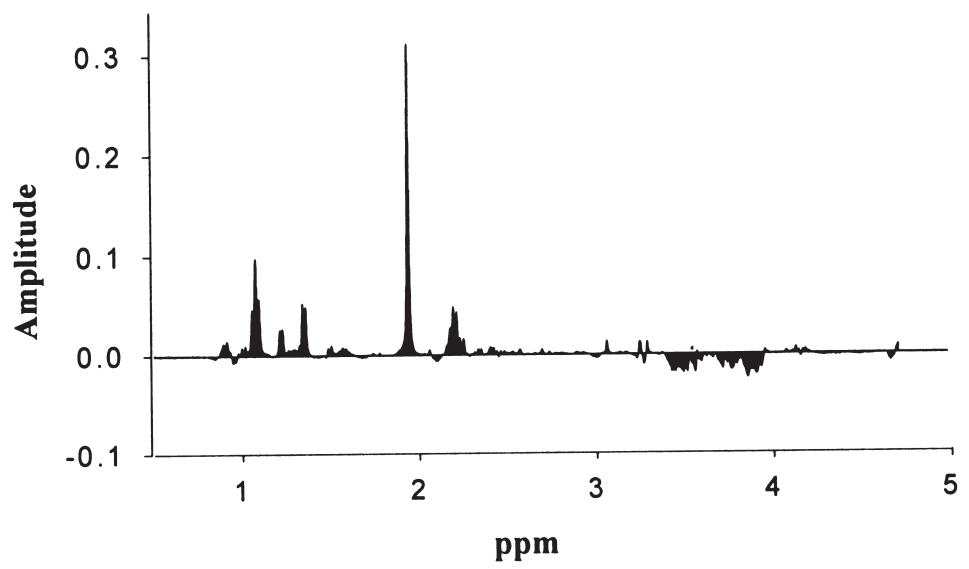

Figure 2. Loading plot. This plot shows elucidation of the resonances in the ${ }^{1} \mathrm{H}$ NMR spectra that are key in discriminating between arterial and portal plasma in PLS-DA. Positive values indicate relatively higher values in portal plasma compared with arterial plasma, whereas negative values indicate lower values

intensity of several spectral regions (1.00-1.10, 1.20-1.23, 1.30-1.37, 1.90-1.97, 2.14-2.26, 3.37-3.60 and 3.68-3.94 ppm). These spectral differences should most probably be ascribed to increased levels of propionate, $\beta$-hydroxybutyrate, lactate, acetate, n-butyrate and decreased levels of glucose in the portal plasma samples, since these metabolites exhibit resonances within these spectral regions (Lindon et al., 1999). It is noticeable that these findings are in agreement with biochemical analyses performed on the plasma samples (Kristensen and Harmon, 2004). The 
obtained results shows that it is possible to detect metabolites in plasma by NMR without time-consuming sample purification. However, before widespread use of the method to quantify concentration differences of metabolites appearing in low concentrations it is necessary to increase the resolution of the spectra and to apply further data analysis in order to use more of the information provided in the spectra.

\section{CONCLUSIONS}

These preliminary results show that a metabolomic approach based on the ${ }^{1} \mathrm{H}$ NMR profile of plasma has potential for investigation of splanchnic metabolism. Further studies exploring the enhancement in spectral resolution associated with a higher magnetic field strength, and studies aiming at making the technique quantitative are needed.

\section{REFERENCES}

Lindon J.C., Nicholson J.K., Everett J.R., 1999. NMR spectroscopy of biofluids. Ann. Rep. NMR Spectr. 38, 1-88

Kristensen N.B., Harmon D.L., 2004. Splanchnic metabolism of VFA absorbed from the washed reticulorumen of steers. J. Anim. Sci. 82, 2033-2042 